\title{
Integrated Support for Location Aware Security Services in Enterprise Wireless Networks
}

\author{
Zhaoyu Liu ${ }^{1}$, Peeyush Sharma ${ }^{2}$, and Jian Raymond $\mathrm{Li}^{3}$ \\ 1 Department of Software Information Systems, \\ University of North Carolina at Charlotte, Charlotte, NC-28223 \\ zhliu@uncc.edu \\ 2 Department of Computer Science, \\ University of North Carolina at Charlotte, Charlotte, NC-28223 \\ psharma3@uncc.edu \\ 3 Cisco Systems Inc., 210 W Tasman Drive, San Jose, CA 95134 \\ rali@cisco.com
}

\begin{abstract}
In wireless computing environments limiting network access to authorized users is paramount for the overall security of network. In addition to basic authentication framework, network access is also governed by the context in which it is being used. In this work, we address security issues based on one such context: location. Location sensitivity is increasingly becoming an integral aspect of wireless and pervasive applications. As user moves around in an ubiquitous environment, access rights and other security services provided to her need to be evaluated accordingly. For such purposes we need a security mechanism that controls the authentication and other security services based on location, in addition to basic identity information. In this paper, we present an architecture of location aware security services for enterprise wireless networks. Our implementation is integrated into the RADIUS system, an Authentication, Authorization and Accounting (AAA) framework. Performace evaluation shows that our implementation is efficient for location based security services.
\end{abstract}

\section{Introduction}

The inception of pervasive computing has allowed a single user to interact with multiple processing devices. Traditional methods used for authentication like password mechanism, do not work sufficiently for security issues in pervasive environment [15]. Moreover it is not always sufficient to authenticate a user only on basis of her identity. In some instances the context of network access assumes more importance than only identification of user [9]. There is a lot of possibilities ranging from providing just the essential security, to providing strong security where same user will have different security services at different locations.

As an example of basic security need, consider Internet services in a coffee shop. It is highly desirable that network access is available only within the shop premises as the shop-owner will prefer to provide it only to customers. On the other side, consider a large enterprise network where personnel in different departments are provided different access rights. The enterprise would ensure that 
basic Internet services are provided in all departments. But information considered confidential for one particular department should not be made available to an employee coming temporarily from another department.

Location authentication alone will not help in ensuring differentiated access rights. For such rights we need a security system where both authentication and authorization are part of the security infrastructure. In this paper, we present an architecture of location aware security services for enterprise wireless networks. Our implementation is integrated into the RADIUS system, an Authentication, Authorization and Accounting (AAA) framework.

The rest of this article is organized as follows. In section 2 we outline the motivation for our work. Section 3 is divided in two parts. The first half describes the working of AAA and the implementation of RADIUS. This discussion is succeeded by our design description in section 3.2. Section 4 covers implementation and performance analysis of integrated support system for location aware security services. And then we discuss the related work in section 5. Section 6 concludes the paper and presents future work of the research.

\section{Background and Motivation}

With wireless computing, we have seen the increasing use of light devices for communication and data processing. Of late, pervasive computing has paved the way for use of smart devices that easily get assimilated in the environment. In the traditional systems access-rights are determined once the credentials submitted by the user confirm with the policies set for her.

In pervasive computing needs are different. One difference lies in the short duration for which a person interacts with a device and then moves on, to access another. The interaction with smart devices is often discrete. If the user has to present her credentials for every smart device then security will become an unwieldy process for the user. The need here is to take the security aspect higher up in hierarchy. This can be achieved by establishing trust between user and environment rather than between a single user and multiple devices in the pervasive environment. This presents need for the context based authentication and access-control. In wireless and pervasive environments one of the major contextual parameters is location. Once a user can be trusted in an environment then smart devices in that environment can be setup according to the preferences of the trusted user. A situation is illustrated below to exemplify this argument.

Consider a classroom that is setup with pervasive devices. Students can use laptops or PDAs to take notes. A webcam is deployed, checking movements to and from the classroom. Multiple smart screens can be used for the course instructions. IR beacons can be used to track the movement of people who keep IR listeners with them. The security task is to associate access rights in this active space with the instructor. This can be done in two ways. Either instructor authenticates to each device in the classroom separately or the space can be setup on the basis of her presence in the smart room; confirmed by IR beacons. Same user can have different authorization levels in different locations. Continuing with the classroom 
scenario, instructor should not get the authority to show the grades of all students in the classroom using the smart screens. Though the instructor has the authority to manage grades of students in her office. In such cases access permissions get determined by the location in which devices are being used.

Furthermore, it is not sufficient to check who is getting network access but necessary to ensure at what level permissions are being granted at various locations. In this work we are presenting the criteria and framework in which location awareness in wireless and pervasive computing can be used to provide varied access rights for user. To ensure a centralized system for security, we are using Remote Authentication Dial In User Service (RADIUS) [1, 14] which is a widely used implementation of AAA.

\section{Location-Aware Architecture}

The Authentication, Authorization and Accountancy (AAA) architecture provides a centralized system where network access can be validated, controlled and monitored. In this work, we are using an AAA implementation, RADIUS, to authenticate user and authorize security services by taking location as a contextual parameter. In the next sub-section we describe basic functionality of RADIUS protocol and then present our location extension design integrated into RADIUS.

\subsection{RADIUS System}

Remote Authentication Dial In User Service (RADIUS) is an AAA access-control protocol originally developed for dial-in services. RADIUS is supported in Virtual Private Network (VPN) and wireless access networks. The basic architecture of RADIUS is depicted in Figure 1. User seeks network access through end user devices. Client takes user's credentials and submits it to the server. RADIUS provides centralized database which contains information needed for user authentication and access-control. This database also keeps a list of configuration items for each user, detailing the type of services user is entitled to receive. The authentication information submitted by user is forwarded to the RADIUS server in form of an access request. This access request contains user's name, her password, client's identification and the port number that user is trying to access.

Upon receiving the access request, RADIUS server first validates the client. If the client does not have the shared secret with the RADIUS server then the request is silently discarded. After successful validation of client the RADIUS server checks a database to find the User-Name. If the User-Name is found in the database then RADIUS checks for User-Password and some other parameters, depending on the configuration setup. In case the User-Password or other configured parameters do not match, an Access-Reject message is sent back to the client.

\subsection{Location Extension into RADIUS}

When RADIUS is used for wireless access, Access Point (AP) works as a RADIUS client. The AP sends the Access-Request to the RADIUS server for any user who 


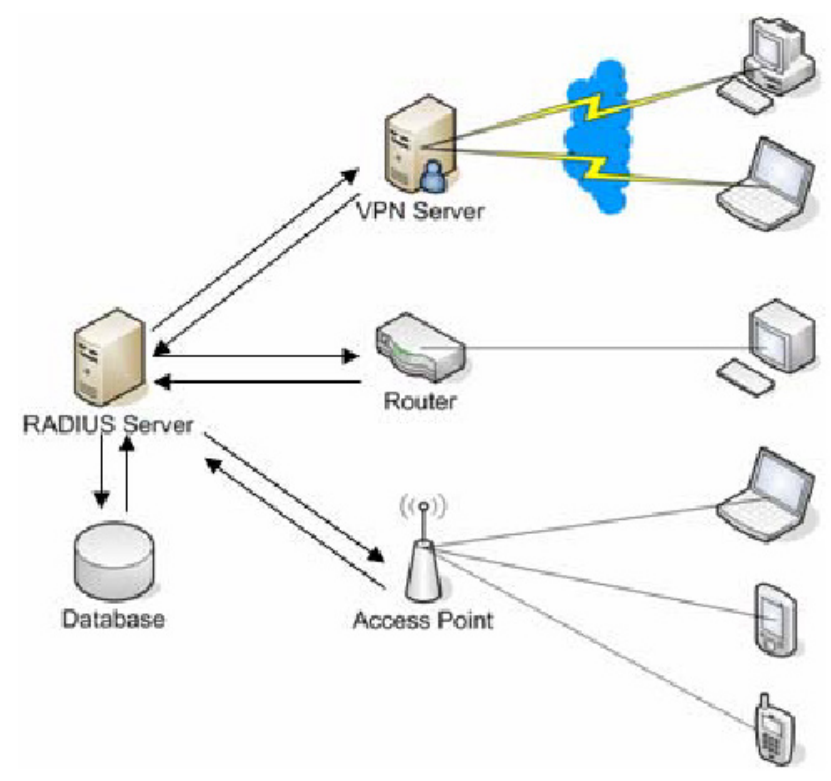

RADIUS server Clients End user devices

Fig. 1. RADIUS Architecture

comes under its coverage and requests access. In a network users are assigned appropriate roles according to level of information they are provided access to, and responsibilities they are accounted for [3]. There are various location aware mechanisms available which can correctly determine the location of a user. One of them is Cricket [6].

In figure 2 the location aware security services framework is shown. The Location Server is used to provide location information to the client. When AP receives the Access-Request from a host in it's vicinity it first contacts the location server. After adding location coordinates to the Access-Request, client forwards request to server. The remote server will have at least one database setup for profile check. When the Server receives request it first authenticates user based on password mechanism set for her in the profile. Authentication is approved if the entry for user in the AA extension suggests that user is allowed to access the network in perimeter specified in the Access-Request. Policies defined in access-control manage authorization levels for users. RADIUS provides security measures for client server communication through MD5 hashing. The security mechanism will be discussed later in section 5.1.

Unified Modeling Language (UML) diagrams can be used to depict sequence of activities $[2,10]$. Events taking place during location aware RADIUS authentication are shown in UML sequence diagram (Figure 3).

The communication scenario can be divided into the following steps. 


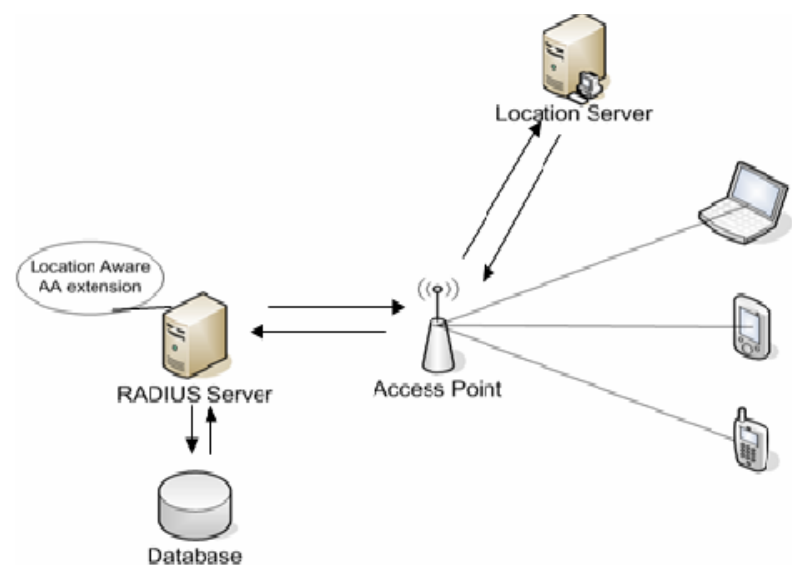

Fig. 2. Location Enhanced RADIUS Server

1. User submits an asynchronous Access-Request to the client.

2. The client checks the user location with the location server.

3. Location server retrieves the current location information for concerned user and sends this information back to the client.

4. Client appends location entities to the original Access-Request.

5. Updated Access-Request packet including location information is forwarded to the RADIUS server.

6. The RADIUS server validates the client. Here we assume that validation succeeds.

7. The RADIUS server searches the database for users profile taking User-Name from the request as a key.

8. If user profile exists for the user then RADIUS retrieves the profile.

9. Configured attributes in user's profile are checked against attributes in the Access-Request (with location information included).

10. Upon verification of request attributes, access is granted to the end user.

In wireless environments users move within the designated wireless network area. To cover such movements security policy is maintained in a hierarchical fashion. Beacons grouped together cover a particular space within the area. User's access rights do not change within a space. But if user moves out of this space then information about access rights is transferred to the higher authority. This authority determines user's location again and grants access rights applicable for that particular location. If user completely moves out of the network area covered by a single centralized authority then credentials have to be resubmitted to the new authority, from the previous authority domain to the new authority domain. If previous and new authorities have trust established between them, then transferred user's credentials can be used to establish proper security services in the new authority domain. 


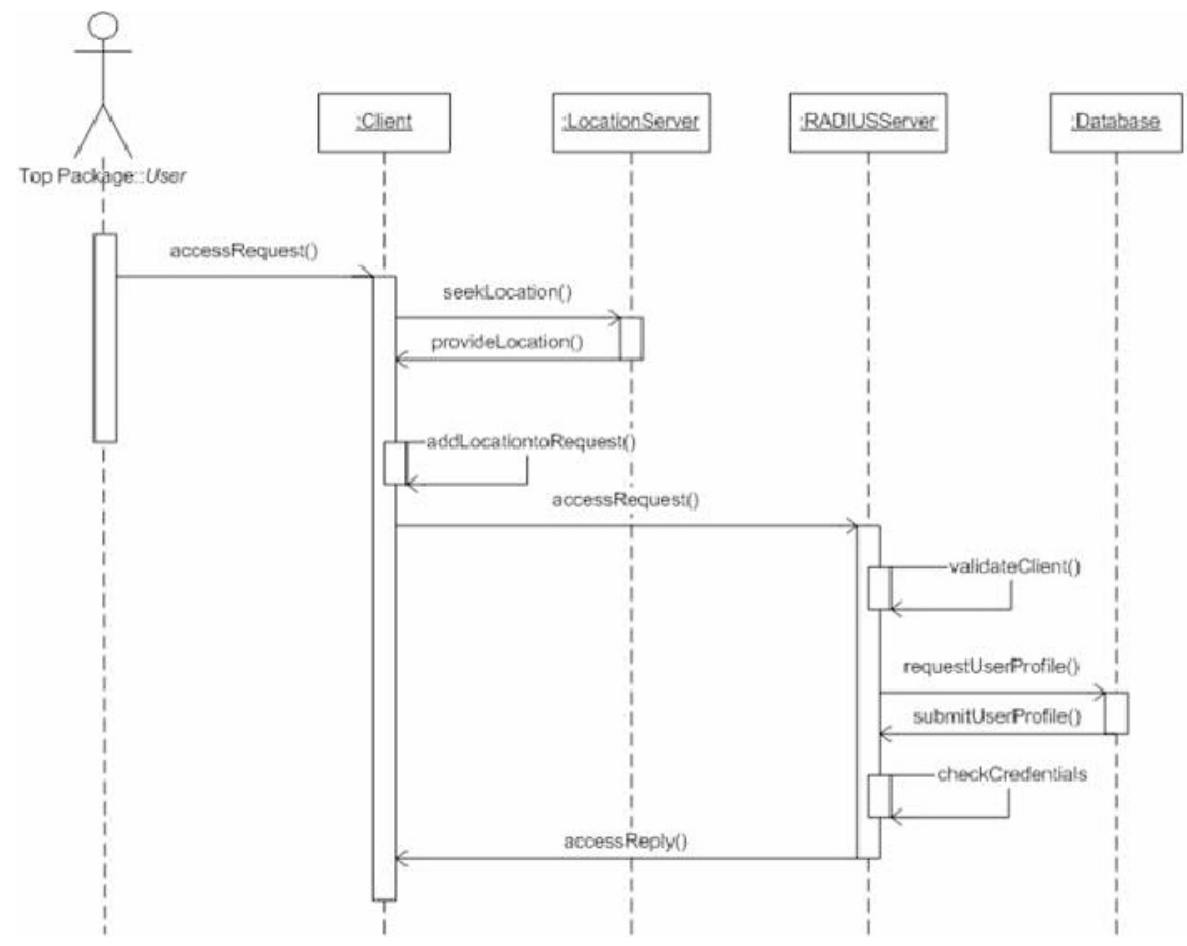

Fig. 3. Sequence Diagram for Location based Authentication

\section{Implementation}

RADIUS has been implemented by various open source software development teams and vendors. For our application we used an open source Software called FreeRADIUS version 1.0.1 on Linux platform Kernel version 2.6.11 [16].

RADIUS server side contains modules to perform configuration updates, database management and other single well-defined tasks. These modules are not needed for the basic application of RADIUS Server but they are used, to perform additional tasks. In our application a location module is added to RADIUS to manage location information. This module manages both authentication and authorization and gets activated only if the configuration for the user has been setup for location based access management. For tracking location information, environment is divided into certain sections or spaces. Location information in the Access-Request contains the space identifier for the section and coordinates for the device in that section. In the following subsections we discuss the security and performance aspects of our implementation.

\subsection{System Security}

RADIUS are vulnerable to real-time active wiretapping attacks [1]. But these attacks can be thwarted by generating unique unpredictable requests. There are two 
kinds of authenticators used in RADIUS. One is request-authenticator which the client uses when sending an Access-Request and the other is response-authenticator used by the server for the Access-Response. A request-authenticator is a MD5 hash generated on a completely random basis by the client and makes the request unpredictable. Once the server receives the Access-Request and decides access for user, it calculates the response-authenticator. This response-authenticator is a MD5 hash based on packet's identification, attributes and request-authenticator. Responseauthenticator is appended to the Access-Response and sent to the client. Since only the client is aware of the random request-authenticator, it can create a MD5 hash of packet's identifiers, attributes and request-authenticator. If such a hash matches with the response-authenticator received from the server then the client can be assured of the integrity of the communication. For our location enhanced service the RADIUS server includes the location information in creating responseauthenticator. Upon receiving response-authenticator from server, client matches it with the MD5 hash created with location information among other identifiers. If two hashes match then the communication is considered to be secured.

\subsection{Performace Evaluation}

Experiments were conducted on the server running on Intel Pentium 4 CPU with a 3.00 GHZ speed and 1.5 GB SDRAM. The client and the location server were also running on the same system. Communication was established through network sockets. Time taken to perform a simple password based authentication was $38 \mathrm{~ms}$ in our setup. The location enhanced authentication mechanism increased this time to $52 \mathrm{~ms}$. The difference in authentication time is attributed to the extra communication link introduced between the location server and the client.

\section{Related Work}

Location awareness has been identified as a key parameter for context aware applications $[8,11]$. Context aware applications are identified as ones which adapt their behavior to changing environments. Determination of user's location and secured exchange of location information form the backbone of such applications.

The work in location awareness in a closed environment has been done by Priyantha et al [6]. They have designed location awareness mechanism termed Cricket for location support for in-building, mobile location dependent systems. Location awareness is achieved through beacons and listeners. Listeners are attached to a mobile device whose location is to be tracked. Beacons are small devices that are spread in the environment. Listener determines it's location on the basis of signals received by various beacons in the region. In our design we use Cricket for location determination. Want et al. [12] have also designed a system for location awareness in closed office environment using active badges.

In [17] Sastry et al. discuss the secure exchange of location information. In [13] author uses address on the Internet as the location parameter. This location information has been used as off-line information to thwart attacks during multi 
party communication. But this off-line information does not cover physical location of user for authentication. In [15] Bardram et al present proximity-based login which allows users to be authenticated on a device just by approaching it physically. But proximity has not been extended as a parameter for authorization. Koo et al. [14] demonstrate use of RADIUS to determine mobile device location.

\section{Conclusion and Future Work}

Location determination applications and toolkits are being deployed widely for pervasive devices in the public networks. With this trend, location awareness mechanisms will have a significant contribution to determining security services. In this paper, we propose an authentacation and access control framework based on location awareness in an enterprise network, as a first step toward pervasive security. The architecture assumes that a location management system can provide users location to the system. The access rights to the user vary according to the policies set for different locations. Our approach is realized by the integration with an AAA framework, the RADIUS system. Enterprise networks with context based security policies will benefit from our system.

In the future work we plan to add support for multiple levels of security. With every authorized service the RADIUS server can determine quality of protection required for secure communication for different locations. Our location module in RADIUS works with several others modules that exist in basic RADIUS architecture. Encryption for different levels of quality of protection can be achieved with security library module working with the location module. Various applications with different security services requirements will be developed based on our system.

\section{Acknowledgements}

This research work is supported by NSF grant 0406325 . We are thankful to the anonymous reviewers for their useful feedback.

\section{References}

1. C. Rigney, S. Willens, A. Rubens, W. Simpson: Remote Authentication Dial In User Service (RADIUS): IETF RFC 2865, June 2000.

2. Eduardo B. Fernandez, Reghu Warrier: Remote Authenticator/Authorizer: In Proceedings of Pattern Languages of Programs (PLoP 2003).

3. David F. Ferraiolo, Janet A. Cugini, D. Richard Kuhn: Role based Access Control (RBAC): Features and Motivations: Proceedings of Computer Security Application Conference, December 1995, pp. 241-248.

4. Narendar Shankar, William A. Arbaugh: On trust for Ubiquitous Computing: In Proceedings of Workshop on Security for Ubiquitous Computing, 2002.

5. Kimmo Janhunen: Recent developments in Authentication, Authorization and Accountancy: 
6. Nissanka B. Priyantha, Anit Chakraborty, Hari Balakrishanan: The Cricket location support system: Proceedings of the 6th ACM MOBICOM, Boston, MA, August 2000.

7. Paramvir Bahl, Anand Balachandran, Allen Miu, Wilf Russell, Geoffrey M. Voelker, Yi-Min Wang: PAWNs: Satisfying the Need for Ubiquitous Secure Connectivity and Location Services: IEEE Personal Communications Magazine (PCS), Vol. 9, No. 1, February 2002.

8. Philippe Debaty, Debbie Caswell: Uniform Web Presence Architecture for People, Places and Things: IEEE Personal Communications 8(4) (August 2001) 611.

9. Tim Kindberg, Kan Zhang, Narendar Shankar: Context authentication using constrained channels: In Proceedings of the Fourth IEEE Workshop on Mobile Computing Systems and Applications (WMCSA 2002), pages 1421, June 2002.

10. Eduardo B. Fernandez, Rouyi Pan: A pattern language for security models: In Proceedings of 8th Conference on Pattern Languages of Programs (PLoP 2001), Monticello, IL, USA, September 2001.

11. Andy Harter, Andy Hopper, Pete Steggles, Andy Ward, Paul Webster: The anatomy of a context aware application: Proceedings of 5th Annual ACM/IEEE International Conference on Mobile Computing and Networking, Seattle, Washington, USA, August 1999, pp. 59-68

12. Roy Want, Andy Hopper, Veronica Falcao, Jonathan Gibbons: The active badge location system: ACM Transactions on Information Systems, vol. 10, pp. 91-102.

13. Riccardo Focardi: Using entity location for the analysis of authentication protocols: In Proceedings of sixth Italian Conference on Theoretical Computer Science (ICTCS 98), Prato, 9-11 November 1998.

14. Simon G. M. Koo, Catherine Rosenberg, Hoi-ho Chan, Yat Chung Lee: Location discovery in Enterprise-based Wireless Networks, Implementation and Application: Second IEEE Workshop on Applications and Services in Wireless Networks (ASWN 2002), Paris. July 2002.

15. Jakob E. Bardram, Rasmus E. Kjaer, Michael Pederson: Context-Aware User Authentication Supporting proximity based login in pervasive computing: Proceedings of Ubicomp 2003: Ubiquitous Computing, volume 2864 of Lecture Notes in Computer Science, pages 107123, Seattle, Washington, USA, Oct. 2003.

16. Linux Kernel 2.6.11: Available at http://www.kernel.org/

17. N. Sastry, U. Shankar, D. Wagner: Secure verification of Location Claims: ACM Workshop on Wireless Security (WiSe 2003), September 2003. 